\title{
Correspondence
}

British Heart fournal, 1977, 39, 227-228

\section{Short-acting and long-acting quinidine for maintenance of sinus rhythm after electrical conversion}

The following two letters have been received regarding the article by Normand et al. (British Heart fournal, 38, 381).

Sir,

Most clinicians like me are happy to use any drug measurable quantitatively in the blood. I was therefore quite amazed to read in the article by Normand et al. that the authors did not mention any quinidine concentration in the blood, an easily obtainable value which is indispensable for the results published. Normand et al. have certainly great faith in the adherence to medical treatment with possible gastrointestinal side-effects in their Parisian countrymen.

\author{
R. J. A. F. Vroom, \\ 101 Aarle-Rixtelseweg, \\ Helmond, \\ Holland.
}

Sir,

Normand et al. concluded that long-acting quinidine preparations are more effective than shortacting quinidine for maintenance of sinus rhythm after electrical conversion of atrial fibrillation. They reasoned that quinidine blood level determinations were not necessary in performing their study. Though this conclusion seems to be a controversial decision, it was at least considered. No consideration was given to the return of mechanical function of the atria after electrical cardioversion. Mahlich et al. (1973) showed that after electrical conversion there might be no return of mechanical function to either right or left atrium, or no return of mechanical function to both atria. The more complete the return of atrial mechanical function the more likely long-term sinus rhythm would follow. Similar correlations of return of mechanical function with maintenance of long-term sinus rhythm after cardioversion for chronic atrial fibrillation were made by Khaja and Parker (1972). Sasse (1974) showed that echocardiography of the posterior left atrial wall could be used to determine the return of left atrial mechanical function after cardioversion, and Mahlich et al. (1973) used kinetocardiography to establish the return of right and/or left atrial mechanical function. Jugular venous pulse tracings may also be used to establish right atrial mechanical function. All these determinations are noninvasive and easy to perform.

It seems that before the efficacy of various quinidine preparations in maintenance of sinus rhythm after cardioversion for atrial fibrillation can be ascertained, the return of mechanical atrial function in differently treated groups of patients must be determined and assigned equally to the groups being compared.

Lewis Sassé,

Department of Internal Medicine,

Southern California Permanente

Medical Group,

1505 North Edgemont Street,

Los Angeles,

California 90027,

U.S.A.

\section{References}

Khaja, F., and Parker, J. O. (1972). Hemodynamic effects of cardioversion in chronic atrial fibrillation. Archives of Internal Medicine, 129, 433-440.

Mahlich, J., Schweizer, W., and Burkart, F. (1973). Atrial function after cardioversion for atrial fibrillation. British Heart fournal, 35, 24-27.

Sasse, L. (1974). Echocardiography of left atrial wall. fournal of the American Medical Association, 228, 1667.

These letters were shown to Dr. Normand and his co-authors who reply as follows.

Sir,

(1) In a previous study, it was shown that the blood quinidine levels obtained with the two preparations are comparable at equal dosage (Renais et al., 1971). With a daily dose equivalent to $0.66 \mathrm{~g}$ quinidine base (as used in our study) the mean quinidine blood level was $3 \mathrm{mg} / \mathrm{l}$, i.e. in the therapeutic range.

(2) As already pointed out in our Discussion (p. 385), previous studies have shown that the blood quinidine level did not differ significantly whether sinus rhythm was maintained or atrial fibrillation 
returned (Byrne-Quinn and Wing, 1970; Hillestad et al., 1971).

(3) Determination of blood quinidine level at a given moment of the treatment did not guarantee the patient's strict adherence to the treatment. Only by measuring the blood quinidine level at the moment of the relapse could it be proved whether or not the relapse was due to an interruption of the treatment.

(4) We took into account the consistence with which the patients followed their treatment and this shows convincingly that the patient's adherence to treatment was much better when long-acting quinidine was prescribed.

In acknowledging Dr. Sassé's comments we should point out that our goal was to compare the efficacy of two quinidine preparations and not to identify patients who are likely to remain in sinus rhythm after electroconversion for atrial fibrillation.

To date there is some evidence that atrial electromechanical dissociation carries a poor prognosis for the maintenance of sinus rhythm (Khaja and Parker, 1972; Mahlich et al., 1973; DeMaria et al., 1975). The fact that in our study long-acting quinidine was more effective than short-acting quinidine in preventing late relapses of atrial fibrillation obviously cannot be explained by a lower incidence of non-restored atrial contraction in the first group for the following reasons:

(1) Patients were assigned at random to short- or long-acting preparation. This offers the best guarantee of similarity between the two groups which in addition was confirmed on 13 points of comparison.

(2) Furthermore it has been shown recently that, in nearly all patients with atrial fibrillation without mitral valve disease - as in our study-cardioversion promptly (within 1 hour) restored effective atrial contraction (DeMaria et al., 1975).

J. P. Normand, J. P. Bourdarias, A. Mathivat, Hôpital Ambroise-Paré, 9 Avenue Charles-de-Gaulle, 92-Boulogne,

France.

\section{References}

De Maria, A. N., Lies, J. E., King, J. F., Miller, R. R., Amsterdam, E. A., and Mason, D. T. (1975). Echographic assessment of atrial transport, mitral movement, and ventricular performance following electroversion of supraventricular arrhythmias. Circulation, 51, 273-282.

Bryne-Quinn, E., and Wing, A. J. (1970). Maintenance of sinus rhythm after DC reversion of atrial fibrillation. A double-blind controlled trial of long-acting quinidine bisulphate. British Heart fournal, 32, 370-376.

Hillestad, L., Bjerkelund, D. D. C., Dale, J., Maltau, J., and Storstein, O. (1971). Quinidine in maintenance of sinus rhythm after electroconversion of chronic atrial fibrillation. A controlled clinical study. British Heart fournal, 33, 518-521.

Renais, J., Scebat, L., and Lenegre, J. (1971). Etude des taux de quinidinémie obtenus par l'administration de quinidine retard. Archives des Maladies du Coeur et des Vaisseaux, 64, 701-711. 